\title{
The Neural Substrate for Binaural Masking Level Differences in the Auditory Cortex
}

\author{
Heather J. Gilbert, Trevor M. Shackleton, Katrin Krumbholz, and Alan R. Palmer \\ MRC Institute of Hearing Research, University Park, Nottingham, NG7 2RD, United Kingdom
}

The binaural masking level difference (BMLD) is a phenomenon whereby a signal that is identical at each ear (S0), masked by a noise that is identical at each ear (N0), can be made 12-15 dB more detectable by inverting the waveform of either the tone or noise at one ear (S $\pi$, $\mathrm{N} \pi$ ). Single-cell responses to BMLD stimuli were measured in the primary auditory cortex of urethane-anesthetized guinea pigs. Firing rate was measured as a function of signal level of a $500 \mathrm{~Hz}$ pure tone masked by low-passed white noise. Responses were similar to those reported in the inferior colliculus. At low signal levels, the response was dominated by the masker. At higher signal levels, firing rate either increased or decreased. Detection thresholds for each neuron were determined using signal detection theory. Few neurons yielded measurable detection thresholds for all stimulus conditions, with a wide range in thresholds. However, across the entire population, the lowest thresholds were consistent with human psychophysical BMLDs. As in the inferior colliculus, the shape of the firing-rate versus signal-level functions depended on the neurons' selectivity for interaural time difference. Our results suggest that, in cortex, BMLD signals are detected from increases or decreases in the firing rate, consistent with predictions of cross-correlation models of binaural processing and that the psychophysical detection threshold is based on the lowest neural thresholds across the population.

Key words: auditory cortex; binaural unmasking; BMLD; hearing

\section{Introduction}

An important sensory function is to detect signals in adverse conditions. In hearing, spatial separation can lead to a dramatic improvement in signal detectability. The binaural masking level difference (BMLD) is often taken as a measure of this ability: a tone signal that is identical at the two ears ( $\mathrm{S} 0)$, masked by a noise that is also identical at the two ears (N0), can be made $12-15 \mathrm{~dB}$ more detectable by sign-inverting either signal or noise at one ear ( $\mathrm{S} \pi$ or $\mathrm{N} \pi$ ) (Hirsh, 1948a, b; Licklider, 1948). The interaural inversion generates interaural time differences (ITDs) not unlike those that arise as a result of spatially separating the signal and noise.

Large BMLDs are only found at low frequencies $(<1.5 \mathrm{kHz})$ and arise from the brainstem machinery sensitive to minute differences in the sound arrival times at the two ears that arise as a result from spatial separation (Jeffress, 1948; Colburn, 1977). Initial attempts to find the neural mechanisms for this unmask-

\footnotetext{
Received March 20, 2014; revised Nov. 4, 2014; accepted Nov. 10, 2014.

Author contributions: H.J.G., T.M.S., K.K., and A.R.P. designed research; H.J.G. performed research; H.J.G. and T.M.S. analyzed data; H.J.G., T.M.S., K.K., and A.R.P. wrote the paper.

This work was supported by the Medical Research Council. We thank Dr Chris Sumner for providing programs for analyzing data; Dr Michael Akeroyd for commenting on the manuscript; Prof. Tino Trahiotis for suggesting the analysis and population plot in Figure 10; and the reviewers for a number of excellent suggestions.

The authors declare no competing financial interests.

This article is freely available online through the J Neurosci Author Open Choice option.

Correspondence should be addressed to Dr. Trevor M Shackleton, MRC Institute of Hearing Research, University Park, Nottingham, NG7 2RD, United Kingdom. E-mail: trevor@ihr.mrc.ac.uk.

DOI:10.1523/JNEUROSCI.1131-14.2015

Copyright (c) 2015 Gilbert et al.

This is an Open Access article distributed under the terms of the Creative Commons Attribution License (http://creativecommons.org/licenses/by/3.0), which permits unrestricted use, distribution and reproduction in any medium provided that the original work is properly attributed.
}

ing in the brainstem and midbrain (inferior colliculus [IC]), by comparing firing rates to an N0 noise and S0 signal (NOSO) with those to the same $\mathrm{N} 0$ noise but an $\mathrm{S} \pi$ signal ( $\mathrm{N} 0 \mathrm{~S} \pi$ ), were largely unsuccessful (Langford, 1984; Caird et al., 1991).

Subsequently, studies in the IC, using signal detection methods, revealed neural responses consistent with the human psychophysically measured BMLDs (McAlpine et al., 1996; Jiang et al., 1997a, b; Palmer et al., 2000; Lane and Delgutte, 2005; Asadollahi et al., 2010). N0 and $\mathrm{N} \pi$ noises drive neurons according to their sensitivity to ITDs. In most neurons, N0 noises drive the neuron well, and S0 signals can be detected by increased firing rate, whereas $S \pi$ signals produce a decrease in firing rate. In contrast, $\mathrm{N} \pi$ noise tends to drive neurons poorly, but the S0 tones are still detected by an increase in firing rate. Masked thresholds for populations of neurons responding to NOS0, $\operatorname{NOS} \pi$, and N $\pi \mathrm{S} 0$ stimuli are consistent with human psychophysics (Hirsh, 1948a, b) in that (1) they show the lowest thresholds near the signal frequency; (2) NOS0 thresholds tend to be greater than N0S $\pi$ and N $\pi S 0$ thresholds; and (3) N0S $\pi$ thresholds tend to be lower than $\mathrm{N} \pi \mathrm{S} 0$ thresholds. It was concluded that different neuron populations contribute to signal detection in different binaural configurations (Jiang et al., 1997a).

Although the IC plays an important role in binaural processing, perception must ultimately depend upon the activity in cortex. Given compelling evidence for widespread convergence from the midbrain into the cortex and extensive feedback loops incorporating the medial geniculate body, encoding of BMLD stimuli in the cortex could be quite different. However, there are few studies of binaural processing between the IC and cortex. Here we apply similar methods as in IC to investigate neural responses to BMLD stimuli in auditory cortex. 
As in the IC, neural responses to BMLD stimuli in auditory cortex were consistent with predictions from binaural crosscorrelation models (for review, see Colburn and Durlach, 1978; Stern and Trahiotis, 1995; Colburn, 1996).

\section{Materials and Methods}

Guinea pigs from an in-house maintained breeding colony (18 male, 16 female weighing between 282 and $974 \mathrm{~g}$ ) were used for the neurophysiological recordings. All experiments were performed under the terms and conditions of licenses issued by the United Kingdom Home Office under the Animals Scientific Procedures Act 1986, project license number 4003049 , and the approval of the ethical review committee of the University of Nottingham.

Surgical procedures. The methods are similar to those used previously (see Jiang et al., 1997a, b). Urethane (0.9-1.3 g/kg in a $20 \%$ solution, i.p.) was used to induce anesthesia. Subsequent analgesia, as determined by suppression of the pedal withdrawal reflex, was maintained with intramuscular injections of $0.2 \mathrm{ml}$ Hypnorm (fentyl citrate $0.315 \mathrm{mg} / \mathrm{ml}$, fluanisone $10 \mathrm{mg} / \mathrm{ml}$, i.m.). Body temperature was maintained at $38^{\circ} \mathrm{C}$ using a rectal probe and a heating blanket (Harvard Apparatus Homeothermic Blanket Control Unit 50787). A premedication of $0.2 \mathrm{ml}$ atropine (600 $\mu \mathrm{l} / \mathrm{ml}$, s.c.) was administered to reduce bronchial secretions. The trachea was cannulated to reduce dead space and allow the animals to be artificially respired with $100 \%$ oxygen throughout the experiment (Harvard Apparatus model 970 ventilator). End-tidal carbon dioxide levels and heart rate (via electrodes either side of the thorax) were monitored (Vetspecs VSM8).

Polythene tubes ( $0.5 \mathrm{~mm}$ inner diameter, $250-400 \mathrm{~mm}$ length) were inserted and sealed into the bullae to allow pressure equalization while maintaining closed field sound presentation. An opening was made in the connective tissue above the foramen magnum to release pressure variations in the cerebrospinal fluid to increase recording stability.

Clear access to the auditory meatus was achieved by removing part of the tragus. The animal was placed into a stereotaxic frame with earbars consisting of hollow perspex speculae so that the tympanic membrane were visible.

The skull overlying the auditory cortex was cleared, and a craniotomy $\sim 6 \mathrm{~mm}$ in diameter was made on the right side to reveal the primary auditory cortex. The dura mater was removed and the exposed cortex covered with agar ( $1.5 \%$ agar in $0.9 \%$ normal saline) to avoid desiccation and to stabilize recordings. Recordings were taken from low-frequency neurons in the primary auditory cortex area A1. In guinea pigs, core area A1 is situated caudal and ventral to the pseudosylvian sulcus (Wallace et al., 2000) with low-frequency units found at the rostral end. Electrophysiological response properties were used to confirm the position of the recording site within the low-frequency area of A1.

Arrays of four glass-coated tungsten microelectrodes were used to record extracellular action potentials (Bullock et al., 1988). These arrays were advanced together using a piezoelectric motor (Burleigh Inchworm IW-700/710).

Stimulus presentation and neuronal recordings were achieved using BrainWare (version 9.19 Jan Schnupp, Oxford University) software and Tucker-Davis Technologies (TDT) hardware.

Neural recording. The action potentials of single neurons were isolated using $50 \mathrm{~ms}$ tone and/or noise bursts as the search stimuli. The raw signals were recorded and bandpass filtered $(0.16-6000 \mathrm{~Hz})$ using a high-impedance headstage (TDT RA16AC) and then digitized (TDT RA16PA). The digitized action potentials were further bandpass filtered $(300-3000 \mathrm{~Hz})$ and amplified using a digital signal processor (TDT RX7), controlled by the BrainWare software. Short portions of the units' action potentials were recorded whenever the amplitude crossed a predetermined threshold, set individually for each unit. These action potentials were analyzed with Plexon Offline Spike Sorter (version 2.8.8). Principal component analysis was used to cluster them into groups that originated from the same neuron. This method allows single-unit activity to be accurately and objectively separated from any multiunit activity.

Stimulus generation. All stimuli were generated with BrainWare using a TDT RX8 Digital Signal Processor, which contains a 24-bit sigma-delta digital-to-analog converter. The signal levels were controlled with programmable attenuators (TDT PA5). The maximum output of the sound system was limited to $\sim 100 \mathrm{~dB}$ SPL. Stimuli were presented either binaurally or monaurally within sealed acoustic systems. All stimuli were gated on and off in software with $2 \mathrm{~ms}$ rise/fall time cosine squared windows (TDT Cos2Gate). The speakers used were custom modified Radioshack 40-1377 tweeters fitted into the hollow speculae via critically damped tubes (diameter $2.5 \mathrm{~mm}$, length $24 \mathrm{~mm}$ : M. Ravicz, Eaton Peabody Laboratory).

The sound system was calibrated at the beginning of each experiment using a $1 / 2$ " Brüel and Kjær 4134 condenser microphone connected to a calibrated $1 \mathrm{~mm}$ probe tube (Brüel and Kjær DB 0241). The end of the probe tube was positioned within the speculum in close proximity to the tympanic membrane. The calibration sound was white noise, presented 20 times. The system response was calculated by Fourier transforming the microphone waveform and correcting for microphone sensitivity and probe-tube characteristics.

Recording paradigms. The $100 \mathrm{~ms}$ tone bursts covering 4 octaves were presented once in pseudo-random order to obtain the neurons' frequency response areas. The tones were presented identically to both ears and repeated once every $400 \mathrm{~ms}$ in $5 \mathrm{~dB}$ steps over a sound level range from 0 to $100 \mathrm{~dB}$ SPL. Spikes were counted within a $10-120 \mathrm{~ms}$ window following the tone onset. The frequency response area is the $2 \mathrm{D}$ pattern of spike counts as a function of frequency and level. Best frequencies were derived offline using the automated method described previously (Palmer et al., 2013; Sumner and Palmer, 2012) with visual confirmation.

Rate-level functions were measured to $100 \mathrm{~ms}$ broadband (50-5000 $\mathrm{Hz}$ ) noise bursts. Like the tones, the noise bursts were presented once every $400 \mathrm{~ms}$ and spikes were counted within a 10-120 ms window after burst onset. The levels covered the range from 0 to $100 \mathrm{~dB}$ SPL in $5 \mathrm{~dB}$ steps. At each level, the stimuli were presented either binaurally or monaurally to left or right ear, yielding three noise rate-level functions for each neuron. The three rate-level functions were obtained simultaneously with all levels for each function presented once in a pseudo-random order before repeating in a different order (100 repeats).

Sensitivity to ITDs was assessed by measuring firing rate as a function of ITD for broadband $(50-5000 \mathrm{~Hz})$ noise and a $500 \mathrm{~Hz}$ tone. The noise was presented at a spectrum level of $23 \mathrm{~dB}$ SPL ( $60 \mathrm{~dB}$ SPL overall), and the tone was presented at $75 \mathrm{~dB}$ SPL. The ITDs ranged from $-2000 \mu$ s to $2000 \mu \mathrm{s}$ in 40 equal steps of $100 \mu \mathrm{s}$. The stimuli were $100 \mathrm{~ms}$ in duration and repeated once every $400 \mathrm{~ms}$. Spikes were counted within 10-120 ms window after stimulus onset. The noise and tone rate-ITD functions were measured separately. For each, all ITDs were presented once in a pseudorandom order before repeating (20 repeats noise, 50 repeats tone).

Masked rate-level functions (MRLFs) were measured to S0 and S $\pi$ signals in the presence of either $\mathrm{N} 0$ or $\mathrm{N} \pi$ masking noise. The signal was a $100 \mathrm{~ms} 500 \mathrm{~Hz}$ pure tone, and the noise was $100 \mathrm{~ms}$ broadband white noise (filtered: $50-5000 \mathrm{~Hz}$ ) with a $23-\mathrm{dB}$ SPL spectrum level. The signal and noise were gated on and off simultaneously and presented once per $400 \mathrm{~ms}$. Spikes were counted within a window $10-120 \mathrm{~ms}$ after stimulus onset. The signal level was varied pseudo-randomly in $5 \mathrm{~dB}$ steps over a range from 0 to $100 \mathrm{~dB}$ SPL. The different interaural configurations of the signal and noise (NOSO, NOS $\pi, \mathrm{N} \pi \mathrm{S} \pi$, and $\mathrm{N} \pi \mathrm{S} 0$ ) were presented in a pseudo-random order. MRLFs were constructed by plotting the total spike count to the signal and noise as a function of the signal level (50 repeats). Poststimulus time histograms (PSTHs) were formed with $1 \mathrm{~ms}$ bins by summing over all tone levels and phases separately for $\mathrm{N} 0$ and $\mathrm{N} \pi$ maskers. Rasters were also plotted across tone level for each of the different interaural configurations of the signal and noise (NOSO, NOS $\pi$, $\mathrm{N} \pi \mathrm{S} \pi$, and $\mathrm{N} \pi \mathrm{S} 0$ ). The PSTHs and raster plots were used to visually determine whether the spike rate exceeded the spontaneous rate at the onset (On), sustained (Sus), or offset (Off) of the response and classify the unit as On, OnOff, OnSus, OnSusOff, Sus, or Off. The variance of spike counts per stimulus as a function of spike count per stimulus were plotted on logarithmic axes and the slope calculated. This gives a measure of how closely the spike generation accords to a Poisson process (variance-mean ratio of 1 ).

Data analysis. The MRLFs were used to determine masked thresholds for the tone using a technique based on signal detection theory (Green 
A

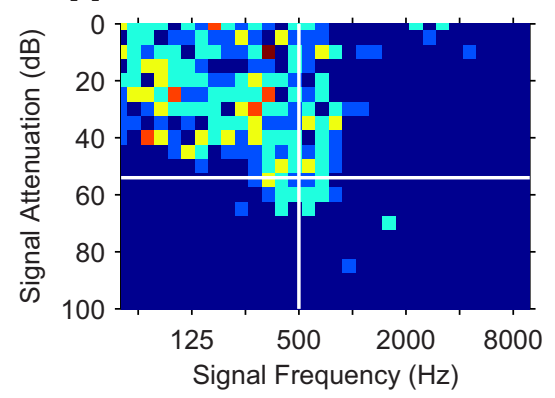

B

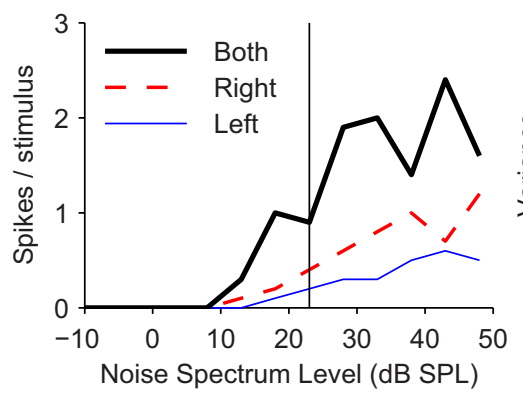

G Slope $0.91 R^{2} 0.93$

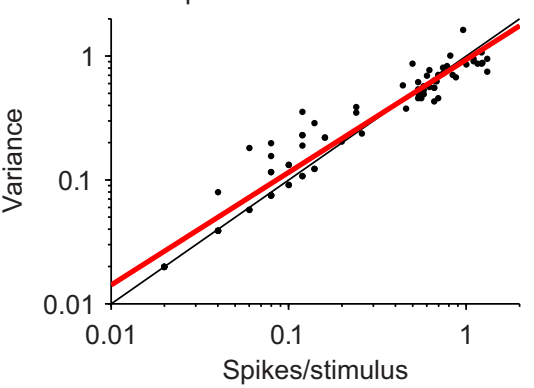

H

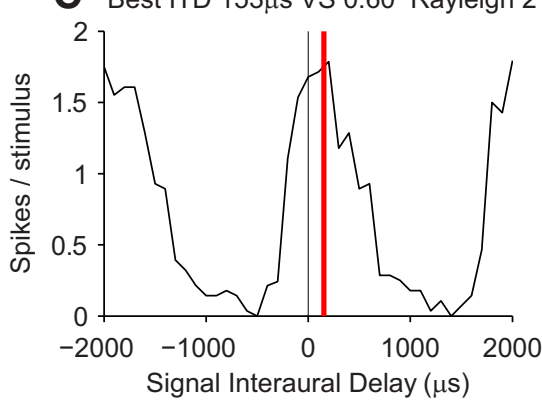

E

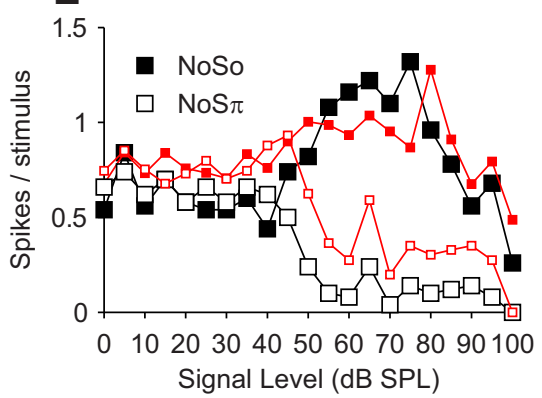

D Peak Tone ITD $100 \mu \mathrm{s}$

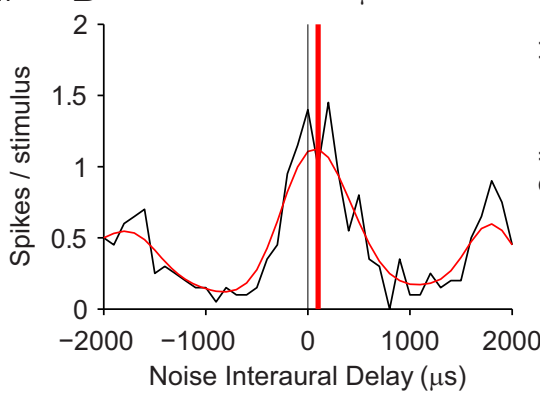

$\mathbf{F}$

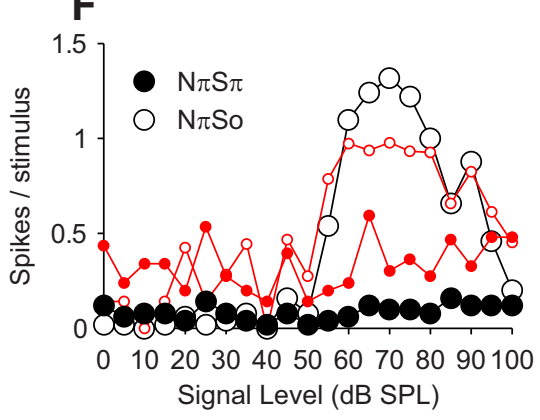

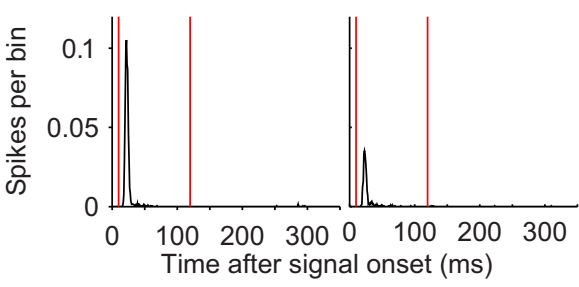

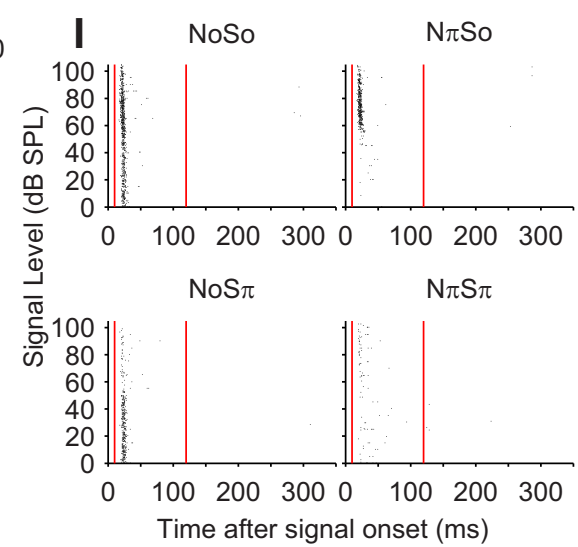

Figure 1. Response profile for a single unit in the primary auditory cortex. $A$, frequency/intensity response area determined with tones presented binaurally with $0 \mu$ s ITD. The maximum tone level is $100 \mathrm{~dB} \mathrm{SPL}( \pm 5 \mathrm{~dB}$ ) across this range. Vertical white line indicates the frequency $(500 \mathrm{~Hz})$ of the tone used subsequently. Horizontal line indicates the level of the noise entering the same auditory filter as the signal. $\boldsymbol{B}$, Rate-level functions for broadband noise presented to the left ear (blue thin line), right ear (red dashed line), or both ears simultaneously (black thick line) at varying sound levels. Vertical line indicates the constant noise spectrum level of $23 \mathrm{~dB}$ SPL used for noise-delay and MRLFs. C, Tone delay function for a $500 \mathrm{~Hz}$ tone presented at $50 \mathrm{~dB}$ SPL. Red vertical line indicates best ITD. $\boldsymbol{D}$, Noise delay function for broadband noise presented at $23 \mathrm{~dB}$ SPL spectrum level. Red curve indicates smoothed noise delay function. Red vertical line indicates best ITD. $\boldsymbol{E}$, $\boldsymbol{F}$, MRLFs for NOSO ( $\boldsymbol{E}$, filled squares), NOS $\pi(\boldsymbol{E}$, open squares), N $\pi \mathrm{S} \pi$ ( $\boldsymbol{F}$, filled circles), and N $\pi \mathrm{SO}$ ( $\boldsymbol{F}$, open circles). Small red symbols represent the corresponding SDs. In each case, a $500 \mathrm{~Hz}$ tone was presented at varying signal levels against a broadband background noise set at a constant level of $23 \mathrm{dBSPL}$ spectrum level. $\mathbf{G}$, Variance of spikes/stimulus versus mean spikes/stimulus pooled across all conditions shown in $\boldsymbol{E}$ and $\boldsymbol{F}$. Diagonal black line indicates equality. Red line indicates the fitted slope. $\boldsymbol{H}$, PSTHs with $1 \mathrm{~ms}$ resolution pooled across all conditions plotted in $\boldsymbol{E}$ (left) and $\boldsymbol{F}$ (right). Red vertical lines indicate the spike counting window. $\boldsymbol{I}$, Raster plots for the conditions shown in $\boldsymbol{E}$ and $\boldsymbol{F}$. Fifty repeats at each signal level are distributed vertically for each sound level increasing vertically. Red vertical lines indicate the spike counting window.

and Swets, 1966). Here, an adapted version of the detectability index $\left(d^{\prime}\right)$ is used, known as the Standard Separation (D) (Sakitt, 1973). This calculation has been previously used to determine masked thresholds for binaural masking conditions in the guinea pig IC and has the advantage of not making any assumptions regarding the underlying distributions (Jiang et al., 1997a, b). At each signal level, l, D is calculated as follows:

$$
D(l)=\frac{r_{N+S}(l)-r_{N}}{\sqrt{s d_{N+S}(l) s d_{N}}}
$$

Here, $r_{N+S}(l)$ is the mean spike rate in response to the noise masked signal at level $l, s d_{N+S}(l)$ is the standard deviation (SD) of the spike rates across different repeats, $r_{N}$ is the mean spike rate across the lowest five signal levels, and $s d_{N}$ is the corresponding SD (calculated across the data for the five lowest levels pooled together).

In previous studies, the masked threshold has been defined as the lowest signal level at which the Standard Separation (D) reached an absolute value of 1.0. This value approximately equates to $75 \%$ correct in a two-alternative-forced-choice psychophysical task. However, in the present study, many MRLFs did not quite reach this criterion value. Consequently, here we used the signal level where $\mathrm{D}$ reached a value of 0.75 as masked threshold, which corresponds to $70 \%$ correct performance in a two-interval forced-choice experiment (Macmillan and Creelman, 2005, their Table A5.7).

Tone rate-ITD functions were analyzed using the method of Goldberg and Brown (1969) to obtain best ITD, vector strength, and Rayleigh statistic. Best ITDs are only reported when the Rayleigh test of uniformity (Buunen and Rhode, 1978) was significant at the 0.05 level $\left(2 n R^{2}>5.991\right.$, where $n$ is the total number of spikes and $R$ is the vector strength).

Noise rate-ITD functions were interpolated to $10 \mu$ s resolution, smoothed with a $300 \mu$ s half-width triangular window, and the ITD of largest peak of the smoothed function chosen as the best ITD. The significance of the peak was tested using bootstrapping. To do this, the responses for every ITD were first randomly redistributed within each repeat (in the experiment, all ITDs were presented before repeating). A simulated noise rate-ITD function was then formed and smoothed as 


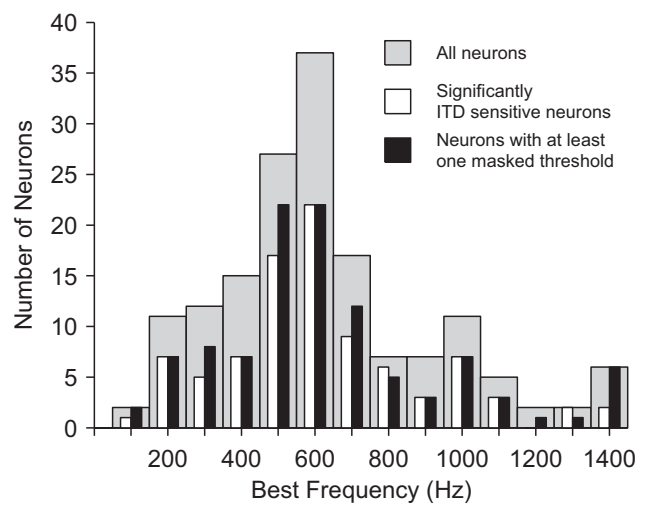

Figure 2. Distribution of best frequencies. Light gray bars represent the distribution of the best frequencies of all 165 units. Black solid bars represent the best frequencies of the 106 neurons that gave measurable thresholds to at least one of the four BMLD conditions. White bars represent the best frequencies of 99 significantly noise ITD-sensitive neurons.
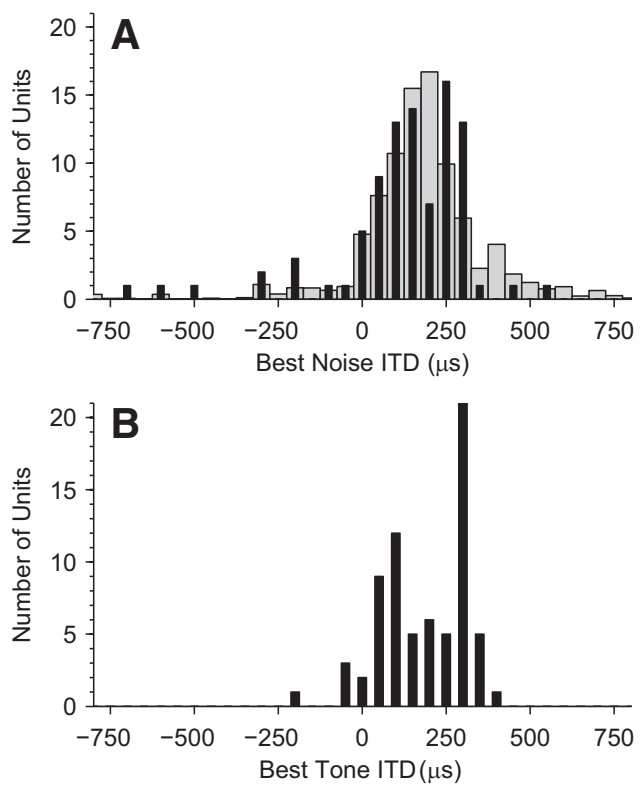

Figure 3. Distribution of significant best ITDs in response to: $(\boldsymbol{A})$ broadband noise and $(\boldsymbol{B})$ $500 \mathrm{~Hz}$ pure tone. Black bars represent best ITDs over an ITD range of $\pm 800 \mu$ s. A, Best ITD calculated as maxima of the smoothed ITD function produced from responses to broadband noise. Only data from the 92 "Peak" units are shown. For comparison, gray bars represent best noise ITDs from the IC, resampled to have the same best frequency distribution as cortical data. B, Best ITD from the "best phase" calculation of Goldberg and Brown (1969) in response to a pure tone signal.

above and the magnitude of the largest peak stored. This was repeated 2000 times to give a distribution of peak heights that would be obtained under the null hypothesis of no tuning to ITD. If the magnitude of the peak obtained from the original data was $>95 \%$ of randomly obtained peaks, then the null hypothesis was rejected and the noise ITD accepted as significant at the 0.05 level.

The distribution of noise best ITDs was compared with that obtained from the IC (McAlpine et al., 2001). Because the IC distribution was shown to be strongly BF-dependent and the distribution of BFs sampled in the IC and cortex were different, the IC data were resampled to have the same BF distribution as obtained in cortex. The data were divided into $100-\mathrm{Hz}$-wide bands and as many samples taken from the IC as occurred in each BF band in the cortex. Sampling was done with replacement. A best ITD histogram was then formed. This procedure was repeated 1000 times to give an average best ITD histogram for IC with the same BF distribution as in cortex. To test whether the best ITD distribu- tion in cortex was different from IC, a $\chi^{2}$ goodness-of-fit test was performed. All bins $<-400 \mu \mathrm{s}$ were pooled, as were all bins $>1000 \mu \mathrm{s}$, to ensure each bin had a non- 0 count. The resampled IC data were treated as the expected distribution, and the cortex data as the measured distribution.

Binaural model. One of the most established models of binaural processing is the cross-correlation model (Colburn and Durlach, 1978; Stern and Trahiotis, 1995; for review, see Colburn, 1996). To compare the predictions of such a model with our physiological data, we implemented a version using a binaural toolbox (M. A. Akeroyd, personal communication). For each ear simulation, signals were first passed through matched gammatone filterbanks before applying a neural transduction process based upon the hair cell model of Meddis et al. (1990) with a high spontaneous rate. Filter outputs from the two ears were then delayed relative to one another to simulate neuron best ITDs. We assumed that all best ITDs are present in all frequency channels. The delayed signals were cross-multiplied, the cross-product integrated over the whole stimulus, and the resulting activity plotted as a function of frequency and best ITD (yielding a type of cross-correlogram, but without normalization; Fig. $12 A-C, F-H)$. The cross-products were averaged across frequencies in the range $300 \mathrm{~Hz}-800 \mathrm{~Hz}$ yielding a summary correlogram showing activity as a function of interaural delay (Fig. 12D,E). The frequencyaveraged binaural cross-products (Fig. $12 D, E$ ) were weighted by the distribution of best ITDs observed in the measured data (Fig. 13).

\section{Results}

Data were obtained from 165 single units in the primary auditory cortex of 34 guinea pigs. Responses were measured to a variety of stimuli to characterize the monaural and binaural responses of each unit and thereby provide explanatory leverage for the BMLD responses. An example of the full range of measurements for each unit is shown in Figure 1.

The frequency response area for this unit is shown in Figure $1 A$. This unit's best frequency was measured as $566 \mathrm{~Hz}$. The signal for the MRLFs was a $500 \mathrm{~Hz}$ tone, and its position with respect to the response area is shown in Figure $1 A$ (vertical line); the level of the masking noise is shown by the horizontal line.

Each unit's response to the noise alone, presented either monaurally to the left or right or binaurally, was measured as a function of noise level (Fig. 1B). These rate-level functions show that this unit is binaurally sensitive: it responds more strongly to noise presented to both ears than to either ear alone, even though the firing threshold is the same for noise presented monaurally or binaurally. The activity of the unit was modulated by the ITD of the signals: the ITD functions for the $500 \mathrm{~Hz}$ tone (Fig. 1C) and the broadband noise (Fig. 1D) both show a clear peak near 0 ITD. The "best ITD" (where the unit responded maximally) was calculated by finding the peak in each ITD function separately (after smoothing). For this unit, the tone and noise ITD functions have best ITDs of 153 and $100 \mu \mathrm{s}$, respectively.

MRLFs were measured in response to the $500 \mathrm{~Hz}$ tone signal against the noise masker in four interaural configurations: N0S0 and $\mathrm{N} 0 \mathrm{~S} \pi$ (Fig. $1 E$ ) and $\mathrm{N} \pi \mathrm{S} \pi$ and $\mathrm{N} \pi \mathrm{S} 0$ (Fig. $1 F$ ). Increasing signal level caused either an increase or decrease in firing rate depending on the stimulus interaural configuration and the best ITD of the unit (for more detail, see later). The rasters for these conditions are shown in Figure $1 I$ and the PSTH pooled across conditions with the same noise phase in Figure $1 H$. In all conditions, this unit fires just after the onset of the signal, and rarely elsewhere, and so was classified as On. The variance of spike counts was proportional to the spike count, with a slope of 0.91 , so firing statistics were close to a Poisson process (Fig. 1G). 
A
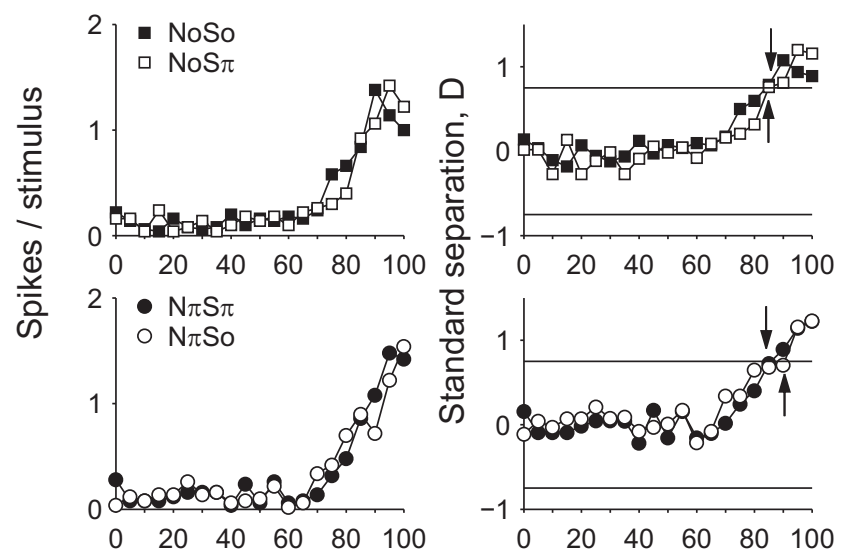

C

Signal Level (dB SPL)

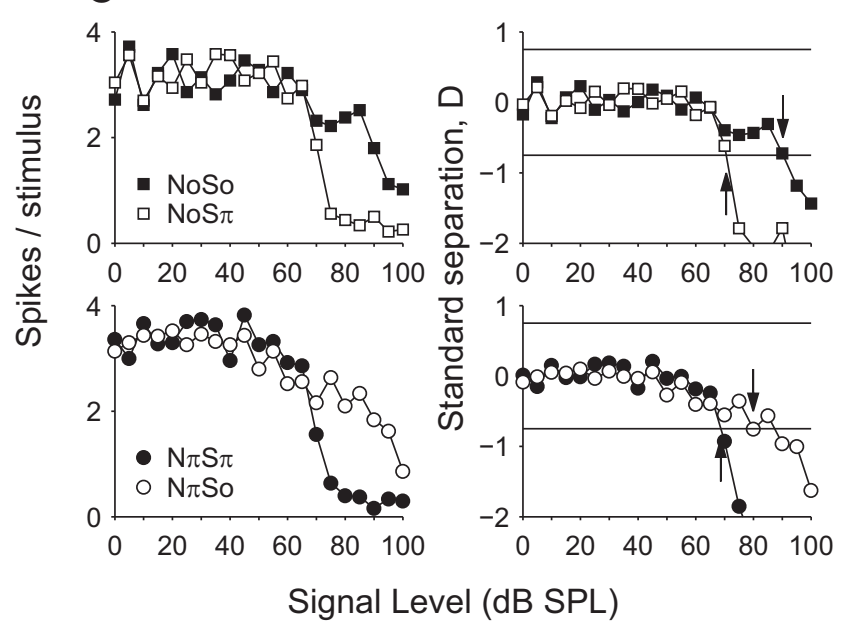

B
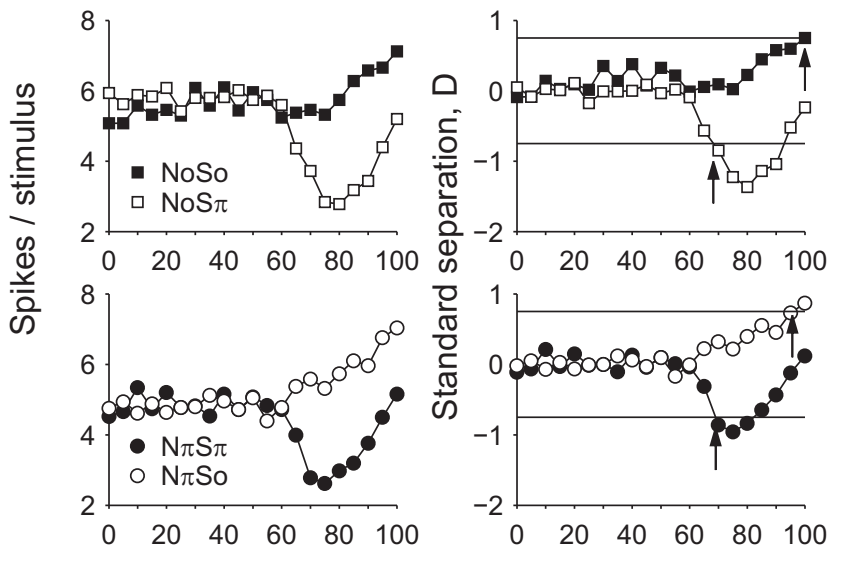

D

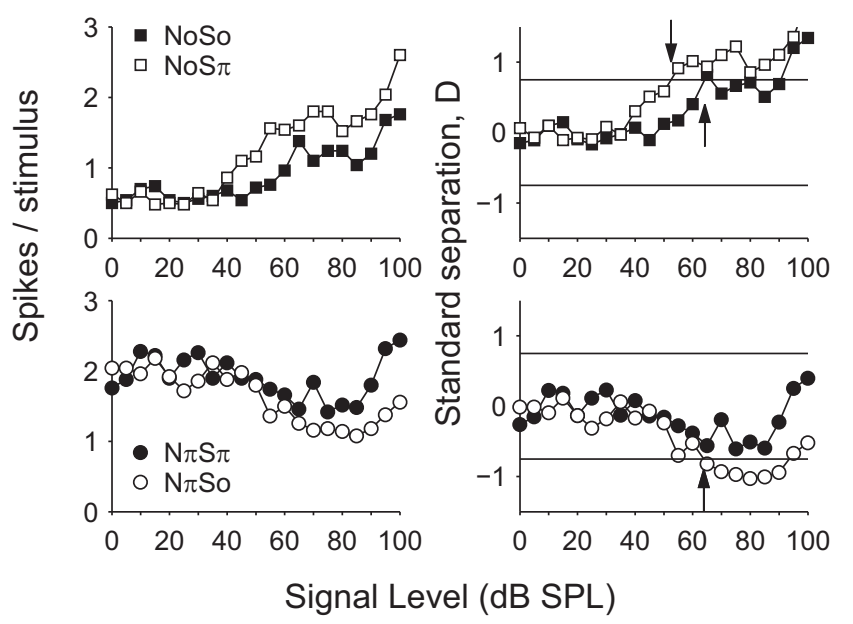

Figure 4. MRLFs and resultant D values for four example neurons. Left of each panel, Masked rate level functions for NOSO (filled squares), NOS $\pi$ (open squares), $N \pi \mathrm{S} \pi$ (filled circles), and $\mathrm{N} \pi \mathrm{SO}$ (open circles). Right of each panel, D (standard separation) functions for the same stimuli. Horizontal lines indicate the $D$ criterion value of \pm 0.75 . Arrows indicate the masked thresholds in each case. $A$, MRLFs and $D$ functions for a unit with $B F$ of $280 \mathrm{~Hz}$ and noise best ITD of $-800 \mu \mathrm{s}$, giving thresholds for all four conditions with an increase in firing rate. $B$, MRLFs and $D$ functions for a unit (BF: $800 \mathrm{~Hz}$; noise best ITD: $200 \mu \mathrm{s}$ ), giving thresholds with an increase in firing rate for NOSO and N $\pi \mathrm{SO}$ and a decrease in firing rate for NOS $\pi$ and N $\pi S \pi$. C, MRLFs and D functions for a unit (BF: 1130 $\mathrm{Hz}$; noise best ITD: $-300 \mu \mathrm{s}$ ), giving thresholds for all four conditions with a decrease in firing rate. $\boldsymbol{D}$, MRLFs and D functions for a unit (BF: $565 \mathrm{~Hz}$; noise best ITD: $-1100 \mu \mathrm{s}$ ), giving thresholds for $\mathrm{NOSO}$ and $\mathrm{NOS} \pi$ with an increase in firing rate, $\mathrm{N} \pi \mathrm{SO}$ with a decrease in firing rate, and no threshold for $\mathrm{N} \pi \mathrm{S} \pi$.

\section{Population characteristics}

The best frequencies of all units included in this study were $<1400 \mathrm{~Hz}$ (range $60-1345 \mathrm{~Hz}$ ), with the majority having best frequencies $\sim 600 \mathrm{~Hz}$ (Fig. 2, gray bars).

The majority of single units recorded (64\%: 106 of 165) gave a measurable masked threshold for at least one of the binaural conditions (Fig. 2, black bars). The majority of units (60\%: 99 of 106) were also significantly sensitive to the noise ITD (Fig. 2, white bars). Because ITD sensitivity and masked thresholds were measured in different runs, it was possible for units that gave a binaural masked threshold and were obviously therefore phase sensitive to produce a nonsignificant noise ITD sensitivity function. There is no difference between the best frequency distributions of these groups.

The firing rate of the units was low, on average 1.0 (1.1 SD) spikes/stimulus in response to N0 noise and 0.62 (0.77 SD) spikes/stimulus in response to $\mathrm{N} \pi$. The majority of units (98\%: 161 of 165) responded at the stimulus onset, 38\% (63 of 165) responded in a sustained manner during the stimulus, and $18 \%$ (30 of 165) responded with a peak at offset. These responses were frequently combined within units; the most common type, how- ever, was On only (52\%: 86 of 165$)$ followed by OnSus ( $28 \%: 46$ of 165) with OnOff (9\%: 15 of 165), OnSusOff ( $8 \%$ : 14 of 165), Sus (2\%: 3 of 165), and Off (1\%: 1 of 165) making up the remainder.

Population distributions of the noise and tone best ITDs are shown in Figure 3 (in black). In line with convention, positive ITDs represent the contralateral, and negative ITDs the ipsilateral, space. For both the noise and tone, the majority of units had best ITDs $<350 \mu$ s within the contralateral hemifield. This is comparable with the range of ITDs calculated from head-related transfer functions (HRTFs) measured acoustically (Sterbing et al., 2003; Greene et al., 2014). Only 7 (7\%) of the 99 delay sensitive units were trough types (Yin and Kuwada, 1983) (i.e., an approximately constant response across ITD except for a dip at the "worst" phase). These were included in the analysis but are not shown plotted in Figure 3.

Also shown in Figure $3 A$ (in gray) is the best ITD distribution for IC neurons previously obtained (McAlpine et al., 2001), resampled so that the BF distribution was the same in IC as cortex (see Data analysis). Each distribution has a peak on the contralat- 
A

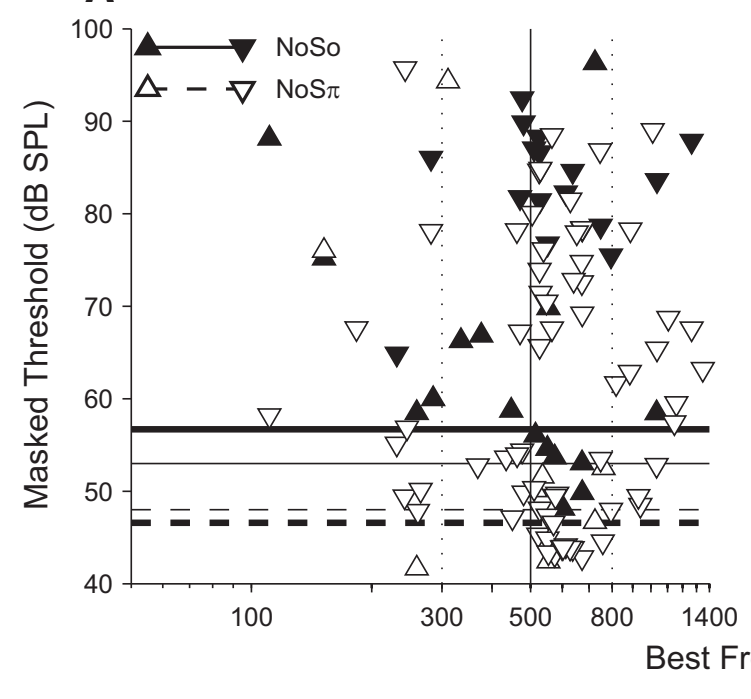

B

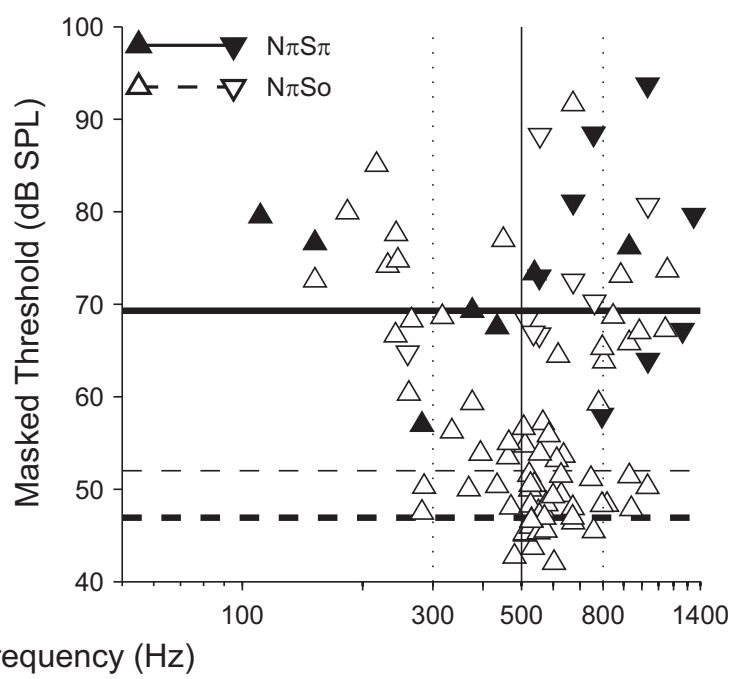

Figure 5. $\quad A, B$, Masked thresholds as a function of neuron best frequency. Masked thresholds for NOSO (filled symbols), NOS $\pi$ (open symbols), $N \pi S \pi$ (filled symbols), and $\mathrm{N} \pi \mathrm{SO}$ (open symbols) plotted against the BF of the neuron. Upward pointing triangles represent $\mathrm{P}$-type thresholds. Downward pointing triangles represent $\mathrm{N}$-type thresholds. Solid vertical line indicates the frequency of the signal $(500 \mathrm{~Hz})$. Dotted vertical lines indicate the BF range over which later analysis occurs $(300-800 \mathrm{~Hz}$ ). Thick horizontal lines indicate the population thresholds (defined as the lowest 25 th percentile over data from 300 to $800 \mathrm{~Hz}$ ) for the current cortical data. Thin line indicates IC data collected previously (there are no N $\pi \mathrm{S} \pi \mathrm{IC}$ data). Solid horizontal lines indicate the homophasic threshold. Dotted horizontal lines indicate the antiphasic threshold.
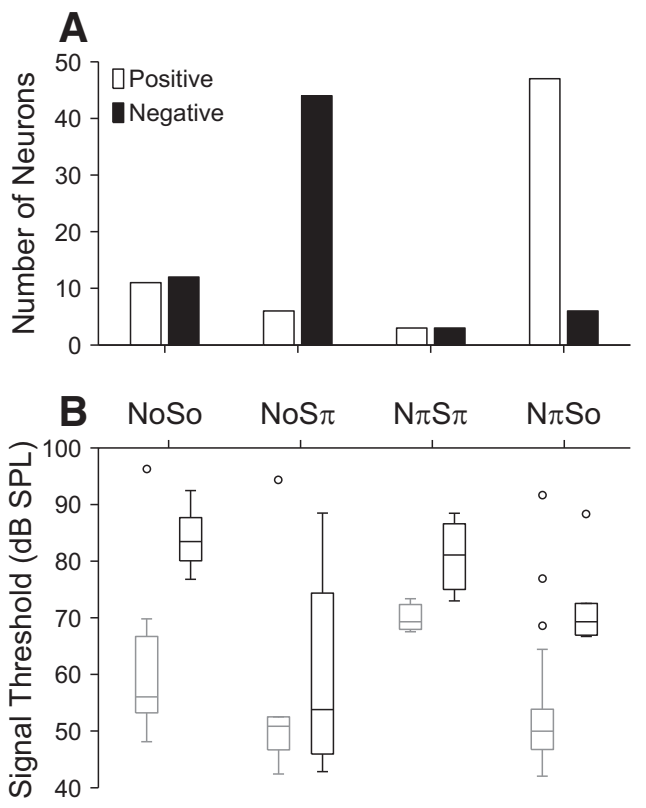

Figure 6. Number of $\mathrm{P}$ - and $\mathrm{N}$-type thresholds obtained for each condition and their average masked thresholds for units with BFs between 300 and $800 \mathrm{~Hz}$. A, Histogram of the number of thresholds determined from increases (Positive D) and decreases (Negative D) in firing rate. $\boldsymbol{B}$, Distributions of masked thresholds separated according to the direction of the change in firing rate: left, positive; right, negative. For each condition, the central mark is the median with the edges of the box being the 25th and 75th percentiles. Whiskers extend to the most extreme points not considered outliers, and outliers are represented as a small circle.

eral side, with fewer units with a best ITD near 0 . The two distributions look qualitatively different; however, the null hypothesis of no difference cannot be rejected ( $\chi^{2}$ goodness of fit, $\chi^{2}=40, \mathrm{df}=28$ ).

\section{Masked thresholds}

Figure 4 shows the MRLFs for all four stimulus configurations in each of four example neurons. As described in detail in Materials

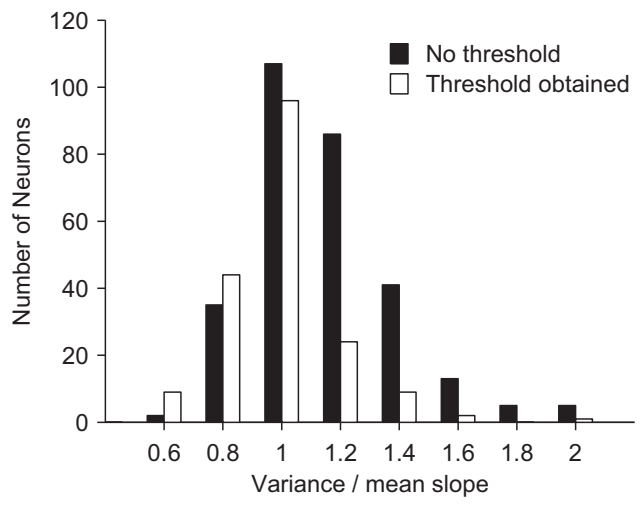

Figure 7. Distribution of variance versus mean spike count slope. Filled bars, Variance/mean slope for masked signal conditions in which no threshold was obtained. Open bars, Variance/ mean slope for masked signal conditions in which a threshold was successfully obtained.

and Methods, the masked threshold is estimated by calculating the standard separation, $\mathrm{D}$, based on the spike rate difference between the signal-plus-noise conditions and the noise-alone condition at each signal level. The signal level at which D reaches a criterion value of 0.75 (in either positive or negative direction; see Fig. 4, horizontal lines) was taken as the unit's threshold for the given condition. In some cases, the MRLFs were nonmonotonic (e.g., Fig. $4 B, D$ ) and therefore met the criterion more than once. When this occurred, the threshold was taken as the lowest signal level at which the criterion was met.

The criterion value was reached by either an increase or decrease in firing rate from the noise alone response. Thresholds based on an increase in firing rate (positive $\mathrm{D}$ values) are referred to as P-type thresholds, whereas those derived from a decrease in firing rate (negative $\mathrm{D}$ value) are referred to as $\mathrm{N}$-type thresholds. Within a single unit, both decreases and increases in firing rate could be observed for different interaural configurations. Of the 106 neurons that yielded at least one measurable masked threshold, only 7 yielded thresholds for all four binaural configurations. 


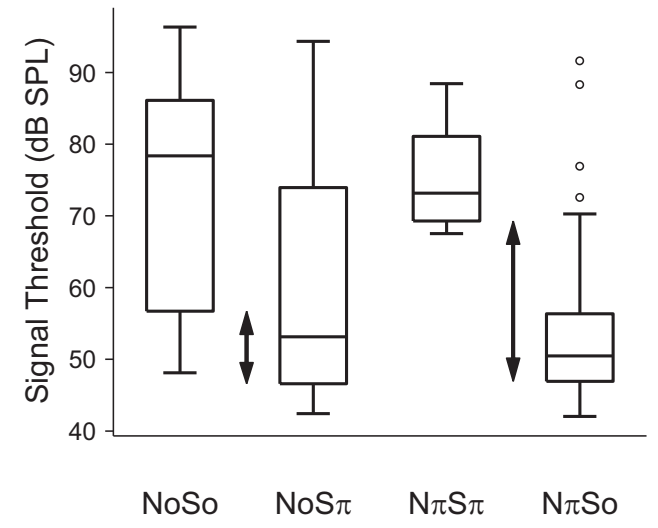

Figure 8. Masked thresholds for all four conditions. Distributions of masked thresholds for units with BFs between 300 and $800 \mathrm{~Hz}$. Arrows indicate the BMLD between NOSO and NOS $\pi$ (left) and $\mathrm{N} \pi \mathrm{S} \pi$ and $\mathrm{N} \pi \mathrm{SO}$ (right) average thresholds calculated from the 25th percentile. For each condition, the central mark is the median with the edges of the box being the 25th and 75th percentiles. Whiskers extend to the most extreme points not considered outliers and outliers are represented as a small circle.

The remaining 99 units were almost equally divided between those giving thresholds for one, two, or three of the interaural configurations. Figure $4 D$ shows an example of a unit for which thresholds could be measured for three of the four conditions. The $\mathrm{D}$ values for $\mathrm{N} \pi \mathrm{S} \pi$ (bottom right, black circle) did not reach the 0.75 criterion, so no threshold was recorded for this condition in this unit. All measurable thresholds were included in the population analysis, not just those where comparable homophasic and antiphasic thresholds were obtained in the same unit.

Masked thresholds were measured from neurons with a variety of best frequencies (Fig. 5). Upward pointing triangles show thresholds for P-type units, and downward pointing triangles for $\mathrm{N}$-type units. There is a wide range of thresholds in all conditions. Unsurprisingly, and in line with previous data, the lowest thresholds were observed in units with best frequencies close to $500 \mathrm{~Hz}$ (Jiang et al., 1997a, b).

Figure 6 shows the number of P- and N-type thresholds for each condition, and the average masked thresholds for each group for units with BFs in the range $300-800 \mathrm{~Hz}$. Substantially more measurable thresholds were obtained for the two antiphasic conditions than for their homophasic counterparts $(n$ : N0S0 $=$ $23, \mathrm{~N} 0 \mathrm{~S} \pi=50, \mathrm{~N} \pi \mathrm{S} \pi=6, \mathrm{~N} \pi \mathrm{S} 0=53$ ). There was also a noticeable difference in the distributions of $\mathrm{P}$ - and N-type thresholds for the two antiphasic conditions. The majority of NOS $\pi$ thresholds were $\mathrm{N}$-type, whereas the majority of $\mathrm{N} \pi \mathrm{S} 0$ thresholds were P-type. P-type responses yielded a lower average threshold for antiphasic than $\mathrm{N}$-type responses; this was generally because the firing rate tended to increase when an signal was added to a noise but, in many conditions, did not reach threshold before the firing rate dropped nonmonotonically and reached the threshold criterion in a decreasing direction.

The wide range of thresholds raises the question of what factors are predictive of threshold. Although many units with BFs close to the signal frequency gave the lowest thresholds, others gave very high thresholds. PSTH response type was not predictive, and the masked thresholds were almost identical whether the response window was constrained to the onset only (10-60 $\mathrm{ms}$ ) or the whole response (10-120 ms). Response windows constrained to the sustained response $(70-120 \mathrm{~ms})$ gave thresholds in 44 units, these tended to be a few $\mathrm{dB}$ higher than to the whole response. Response windows constrained to the offset response
(120-170 ms) gave thresholds in 20 units, these were up to $20 \mathrm{~dB}$ higher. There were small effects of noise only firing rate and the $\mathrm{SD}$ of noise only firing rate (where the noise only response was defined as the bottom $20 \mathrm{~dB}$ of the MRLF). Neither of these measures had an effect on the homophasic thresholds but were barely significant ( $t$ test, $p<0.05$ ) in the antiphasic conditions. Here units that gave a measurable masked threshold tended to have a lower firing rate and SD than those which gave no measurable masked threshold. However, although just significant, the effect was barely noticeable and is not illustrated. Unsurprisingly, the maximum rate across the MRLF was significantly ( $t$ test, $p<0.01)$ related to the ability to measure a masked threshold. This simply reflects the fact that units with a large dynamic range in response to the masked stimulus were able to cross threshold.

The standard separation, $\mathrm{D}$, is a combination of two factors, the SD and the difference in firing rate between noise alone and noise plus signal conditions. The variation in one or both can determine threshold (Shackleton et al., 2003; Tollin et al., 2008). In a Poisson process, the variance is proportional to the firing rate, so they are not independent of each other. We measured the slope of the log-log plot of variance against spike count separately for each binaural masking condition (N0S0, NOS $\pi, \mathrm{N} \pi \mathrm{S} \pi$, and $\mathrm{N} \pi \mathrm{S} 0$ ) as firing rate varied with signal level. The distributions were independent of condition so are plotted pooled together in Figure 7. The distribution is centered on one, suggesting that most units had nearly Poisson firing statistics. Two distributions are plotted depending upon whether a masked threshold was obtained (white bars) or not (black bars). Although the difference is slight, the distribution is biased toward lower slopes when a threshold was obtained ( $t$ test, $p<0.05$ for N0S0, N0S $\pi$, and $\mathrm{N} \pi \mathrm{S} 0$ ). This means that variance did not increase as rapidly as firing rate as firing rate increased. In conclusion, firing rate and the SD of firing rate are proportional to each other in these masked signal conditions, and each factor is almost equally important; however, for some units, the SD is lower than predicted from Poisson statistics, and this favors lower thresholds.

\section{BMLDs}

The relatively low number of homophasic thresholds measured means that intraunit BMLDs could only be calculated in a small number of cases (29 for N0S0 vs N0S $\pi, 10$ for $\mathrm{N} \pi \mathrm{S} \pi$ vs N $\pi \mathrm{S} 0$ ). The majority of the single units for which both homophasic and antiphasic thresholds could be determined showed positive BMLDs, with antiphasic thresholds lower than homophasic thresholds (37 of 39). Many of the intraunit BMLDs observed here, however, were much larger than is conventionally found in psychophysical experiments (12-15 dB): 20 units had BMLDs $>20$ $\mathrm{dB}$. These very large BMLDs were due to the units showing very little sensitivity to the homophasic condition, resulting in an unusually high threshold for that condition.

A number of neurons in the population $\sim 500 \mathrm{~Hz}$ had a very low sensitivity to certain interaural configurations, with the $\mathrm{D}$ values only reaching the criterion at very high signal levels. An overall mean of all thresholds for each condition would therefore give a population estimate biased toward these very high thresholds. However, it is plausible that the determination of the psychophysical threshold would be based upon the responses of the most sensitive neurons in the population (Jiang et al., 1997a, b; Parker and Newsome, 1998; Palmer et al., 2000). For this reason, the population threshold was defined as the lower quartile of the thresholds (25th percentile) measured for neurons with best frequencies between 300 and $800 \mathrm{~Hz}$ giving thresholds of N0S0 $=57$ 
$\mathrm{dB}, \mathrm{N} 0 \mathrm{~S} \pi=47 \mathrm{~dB}, \mathrm{~N} \pi \mathrm{S} \pi=69 \mathrm{~dB}$, and $\mathrm{N} \pi \mathrm{S} 0=47 \mathrm{~dB}$. The choice of $25 \%$ was to a large extent arbitrary; it was chosen to be a more robust population estimate than an extreme value and to correspond to the quartile plotted by default in "box-plots." These thresholds are plotted as horizontal lines in Figure 5. The corresponding thresholds in the IC were N0S0 $=53 \mathrm{~dB}$, N0S $\pi=48 \mathrm{~dB}$ (Jiang et al., 1997a), and in a later series of experiments measured at a $10 \mathrm{~dB}$ higher noise spectrum level: N0S0 $=57 \mathrm{~dB}, \mathrm{~N} 0 \mathrm{~S} \pi=52 \mathrm{~dB}$, and $\mathrm{N} \pi \mathrm{S} 0=56 \mathrm{~dB}$ (Palmer et al., 2000). $\mathrm{N} \pi \mathrm{S} \pi$ was not measured and $\mathrm{N} 0 \mathrm{~S} \pi$ not shown in the paper. It is not entirely clear why these thresholds are not proportional to masker level, appearing only $4 \mathrm{~dB}$ higher, but it is known that psychophysically BMLDs vary with overall masker level (Durlach and Colburn, 1978). Applying this correction gives a comparison IC threshold of $\mathrm{N} \pi \mathrm{S} 0=52 \mathrm{~dB}$. In cortex, the antiphasic threshold is lower and the homophasic threshold higher than in the IC. The overall higher homophasic thresholds may be due to a more restricted firing rate range in cortex than IC. The very high homophasic thresholds tended to be due to nonmonotonic MRLFs, which started in one direction as signal level increased but did not reach threshold before they turned and reached threshold in the opposite direction. Antiphasic thresholds tended to be reached in the monotonic portion of the masked ratelevel curve.

The box-plot summary of the thresholds of neurons with best frequencies between 300 and $800 \mathrm{~Hz}$ is shown in Figure 8 $(n: \mathrm{N} 0 \mathrm{~S} 0=23, \mathrm{~N} 0 \mathrm{~S} \pi=50, \mathrm{~N} \pi \mathrm{S} \pi=6$, $\mathrm{N} \pi \mathrm{S} 0=53$ ), and the binaural masking release between the 25th percentiles is shown as arrows. The binaural masking release effect observed psychophysically is replicated here. In both the NOSO-NOS $\pi$ and $\mathrm{N} \pi \mathrm{S} \pi-\mathrm{N} \pi \mathrm{S} 0$ comparisons, the homophasic threshold is higher than the antiphasic threshold, giving BMLDs of 10 and $22 \mathrm{~dB}$, respectively (Fig. 8, arrows).

\section{Effect of best ITD on responses to BMLD stimuli}

To relate the response to the BMLD stimuli to the neurons' selectivity for ITDs, we computed the MRLFs for all neurons with best frequencies between 300 and $800 \mathrm{~Hz}$ summed across neurons with noise best ITDs in $100 \mu$ s bins. Examples of these summed masked rate level functions are shown in Figure 9 for each of the binaural masking configurations and six best ITD bins between $\pm 300 \mu \mathrm{s}$. We also show these data as the distribution of activity across best ITD for three different tone levels and for noise alone (Fig. 10). The three signal levels and the noise alone are indicated in Figure 9 (vertical lines; the black noise alone line is near the

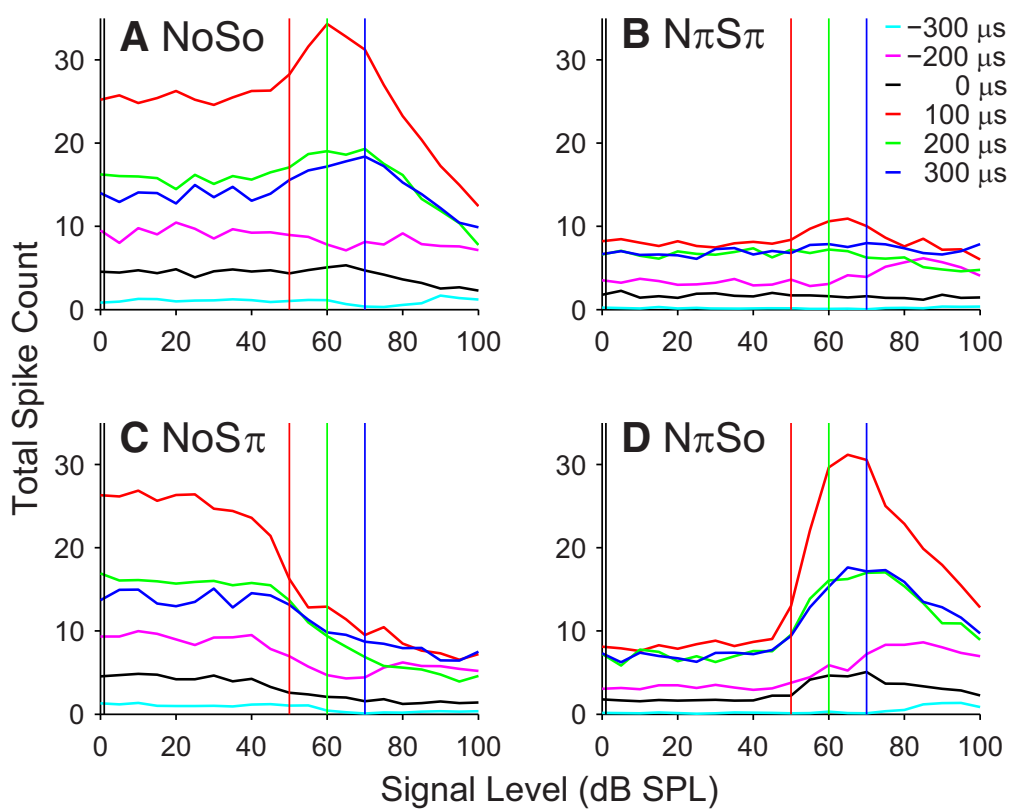

Figure 9. $A-D$, Summed masked rate level functions for neurons with BFs $\sim 500 \mathrm{~Hz}$ and for different noise best ITDs. The key indicates the best noise ITD values. Each point in each curve is a sum of the firing rate at that sound level of all units with BFs between $300 \mathrm{~Hz}$ and $800 \mathrm{~Hz}$ and best noise ITD as indicated. Vertical lines indicate the sound levels used to compute the population responses shown in Figure 10 with the same color coding as Figure 10.

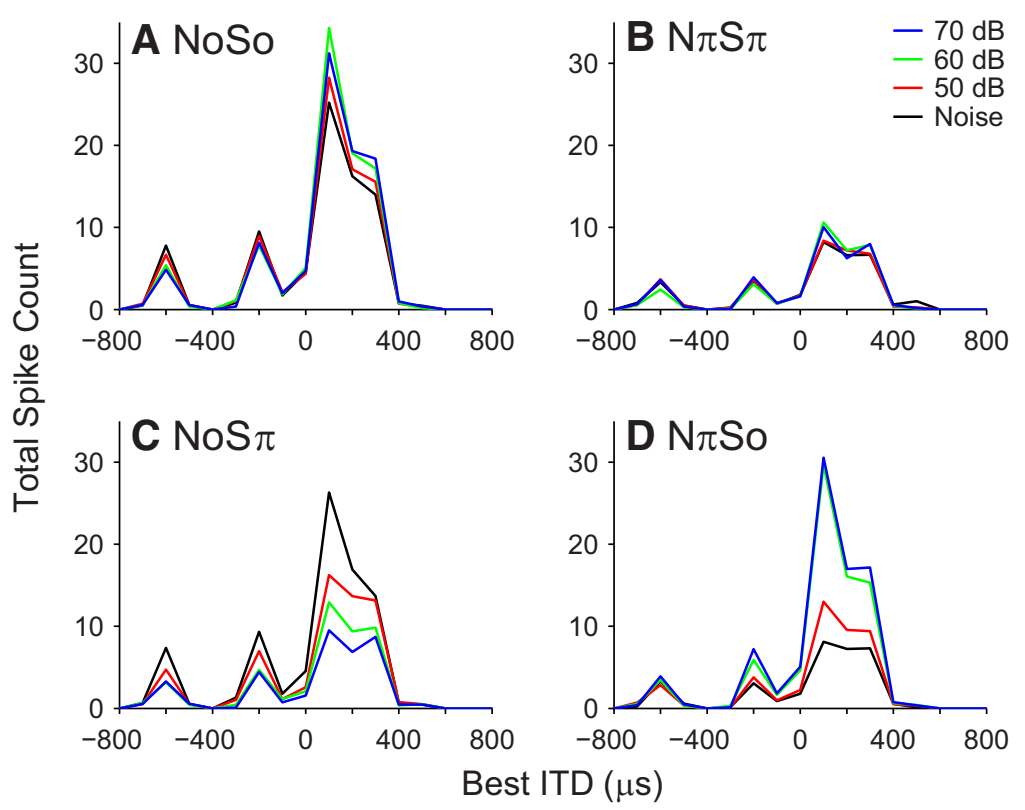

Figure 10. $\quad \boldsymbol{A}-\boldsymbol{D}$, Total neuronal response to four signal levels as a function of best noise ITD of the unit. Black line indicates unit responses to the noise alone (either N0 for $\boldsymbol{A}$ and $\boldsymbol{C}$ or $\mathrm{N} \pi$ for $\boldsymbol{B}$ and $\boldsymbol{D}$ ). The red, green, and blue lines are the summed neuronal responses at increasing signal levels (see key).

ordinate). The three signal levels range upward in $10 \mathrm{~dB}$ steps from near the estimated antiphasic population threshold.

The general shapes of the summed MRLFs in Figure 9 follow those of individual single-neuron MRLFs (compare with Fig. 4) and are predictable from the rate-ITD functions: the closer the best ITDs were to 0 , the greater the response to interaurally identical stimuli (N0, S0) and the smaller the response to interaurally inverted stimuli $(\mathrm{N} \pi, \mathrm{S} \pi)$. As a result, neurons within best ITD bins close to 0 tended to show a decrease in response with increasing signal level in the NOS $\pi$ condition, but an increase in the 

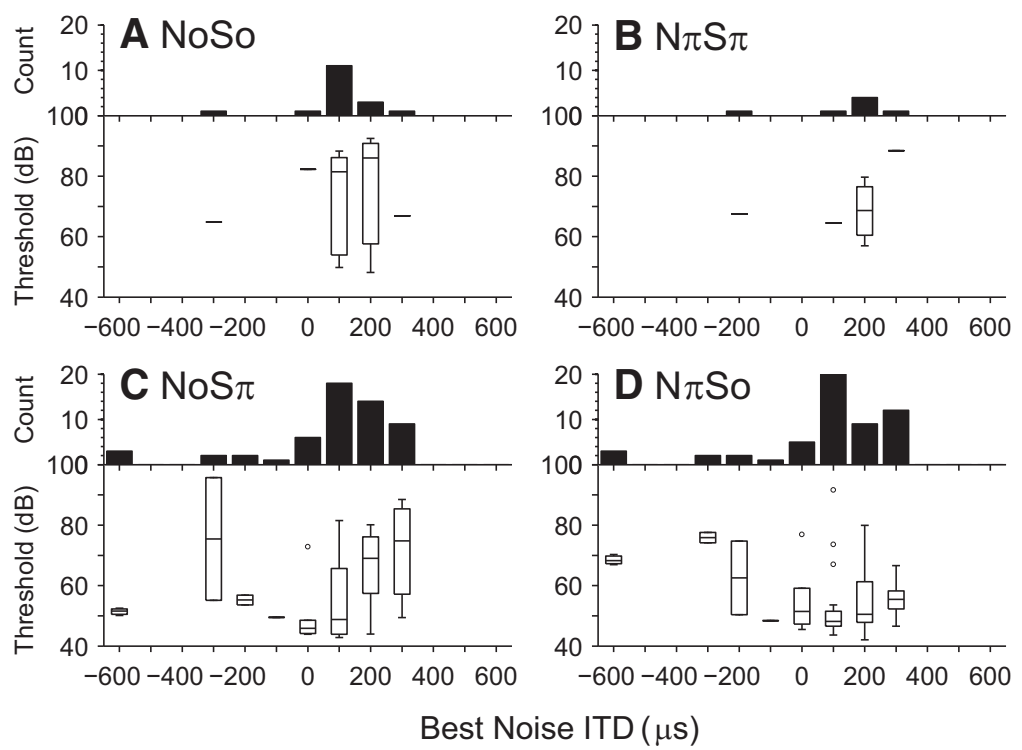

Figure 11. Masked thresholds and number of thresholds obtained for each condition as a function of the noise best ITD of the unit. Data are for the different binaural masking conditions: $\boldsymbol{A}, \mathrm{NOSO}, \boldsymbol{B}, \mathrm{N} \pi S \pi ; \boldsymbol{C}, \mathrm{NOS} \pi ; \boldsymbol{D}, \mathrm{N} \pi \mathrm{SO}$. Bottom, Distributions of masked thresholds separated according to best noise ITD for units with a significant noise ITD function. For each condition, the central mark is the median with the edges of the box being the 25 th and 75 th percentiles. Whiskers extend to the most extreme points not considered outliers, and outliers are represented as a small circle. Top, Histogram of the number of units with significant noise ITD functions which make up the threshold distribution.

$\mathrm{N} \pi \mathrm{S} 0$ condition. As the signal level was increased $>60-70 \mathrm{~dB}$ SPL, the total response of these neurons decreased due to the large proportion of nonmonotonic MRLFs measured for NOS0 ( $\sim 40 \%$; see, e.g., Fig. $1 E$ where the firing rate at $80 \mathrm{~dB}$ is on the descending part of the nonmonotonic function).

As shown in Figure 10, the effect of increasing the signal level can also be observed for each of the four interaural configurations across the range of best ITDs. For NOS0, increasing the signal level increased the total response slightly for neurons tuned to 100, 200 , and $300 \mu$ STD. In the N0S $\pi$ condition, the overall response of neurons with ITDs within a few hundred microseconds of zero in the contralateral hemifield decreased monotonically with increasing signal level. In the $\mathrm{N} \pi \mathrm{S} \pi$ condition, an increase in signal level had almost no effect on the overall responses at any best ITD. This presumably is a floor effect: $\mathrm{N} \pi$ is at the least optimal ITD for these units; hence, the baseline firing is low and the S $\pi$ signal has little effect in changing this rate. However, a large change was observed in the responses of neurons with best ITDs near 0 with the introduction of the S0 signal to $\mathrm{N} \pi$ noise (Fig. $10 D)$. The low baseline rate in response to the $\mathrm{N} \pi$ noise is increased markedly by increasing levels of the S0 tone.

The representations of the neuronal responses shown in Figure 10 are highly weighted toward the most commonly measured best ITDs (50-350 $\mu \mathrm{s})$; very few neurons in the present dataset had best ITDs outside of this range, which corresponds to the measured range of ecologically available ITDs (Sterbing et al., 2003; Greene et al., 2014).

As explained above, we would expect neural responses to be most different between in-phase and out-of-phase stimuli when the best ITD of the neuron is close to zero simply because the in-phase stimulus is at the peak ITD sensitivity and the out-ofphase at the trough. If the best ITD of the neuron is away from zero, then the responses to in-phase stimuli will be reduced and those to out-of-phase stimuli increased, so there is less modulation in response. We might, therefore, expect that antiphasic masked thresholds would be lower for neurons with best ITDs near 0 compared with those further from zero because there is more scope for the firing rate to be modulated by the signal. To test this idea, we plotted the masked threshold as a function of noise best ITD (Fig. 11) for each binaural condition. Fig. 11 (bottom) shows a box-plot of the thresholds at each best ITD; Fig. 11 (top) shows the number of neurons that contributed. It can be seen that, although there is a great deal of variability, both the median and threshold (lower quartile) for the antiphasic conditions are lowest near 0 best ITD.

\section{Discussion}

A priori it might be expected that there would be significant modification of the coding of BMLD stimuli in the medial geniculate body, between IC and cortex, and within the cortex because the activity in A1 is known to be modulated depending upon stimulus history and attentional state (e.g., Lee and Middlebrooks, 2013; Yin et al., 2014). However, the similarity of our cortical BMLD measurements to human psychophysical measures suggests that there may be no need for further enhancement by top-down influences.

\section{Effect of anesthesia and recording position}

The measurements reported here used the same anesthetics as similar measurements in the IC. However, some anesthetics may have a differential effect at the cortex compared with the IC (TerMikaelian et al., 2007), particularly on the temporal aspects of the responses. They used pentobarbitone and ketamine, which both have effects on specific ion channels (MacDonald et al., 1989; Franks and Lieb, 1994). We used urethane, which has been shown to induce anesthesia by an effect across a range of ion channels rather than specific families (Hara and Harris, 2002). We supplement with hypnorm, a combination of an opioid and a sedative. This regimen is likely to have different effects to pentobarbitone and ketamine, but we are unaware of data that address differential effects of these drugs at IC and cortex. Our measurements do not depend on precise timing of spikes (all fine timing information about ITDs is converted to mean discharge rates at the level of the brainstem), and so, may not be subject to such differential effects of the anesthetic, even if they occur with this regimen.

We did not histologically verify the recording position; however, past experience suggests that these recordings were mostly obtained in layers III and IV of AI. These are the input layers to the cortex. It is therefore possible that a large amount of processing occurs within the cerebral cortex after these recordings.

\section{Population measures}

The range of best frequencies observed here is biased by the deliberate selection of the recording sites in the low-frequency part of AI and is comparable with those measured in the IC in similar studies in the same species (Jiang et al., 1997a, b; Palmer et al., 2000). In the IC, the best ITD distribution was highly best frequency-dependent, so the IC data were resampled to have the 
A No
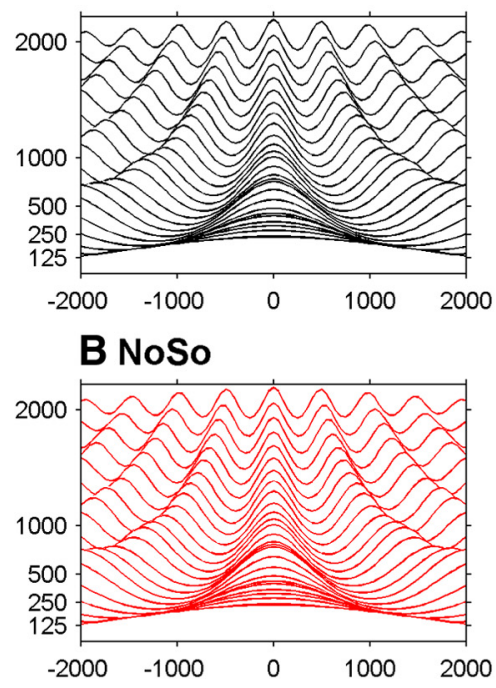

$\mathrm{C}$ NoS $\pi$

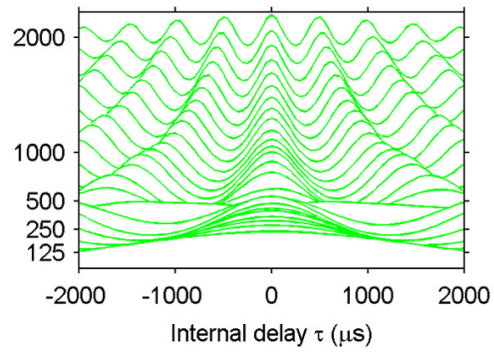

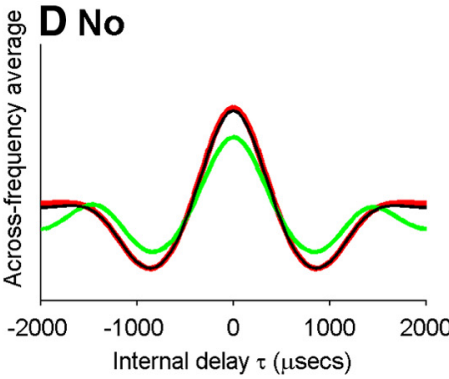

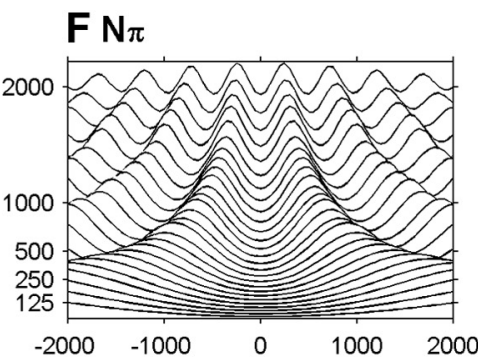

$\mathbf{G} \mathbf{N} \pi \boldsymbol{S} \pi$

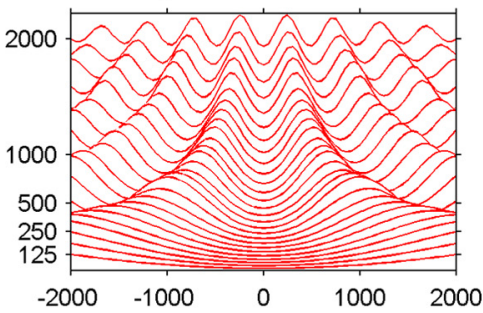

$\mathrm{H}$ N $\pi$ So

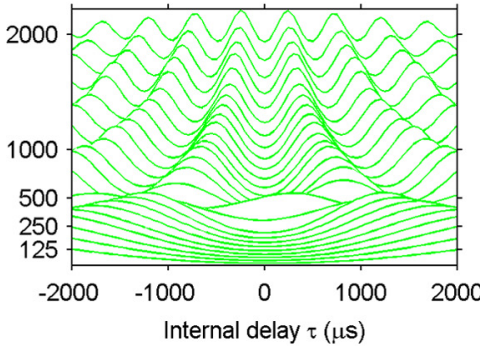

Figure 12. Cross-correlation model of BMLD. Left and right columns represent $3 \mathrm{D}$ correlogram for both noise alone $(\boldsymbol{A}, \mathrm{NO} ; \boldsymbol{F}, \mathrm{N} \pi)$ and signal masked by noise $(\boldsymbol{B}, \mathrm{NOSO} ; \boldsymbol{C}, \mathrm{NOS} \pi ; \boldsymbol{G}, \mathrm{N} \pi \mathrm{S} \pi ; \boldsymbol{H}$, $\mathrm{N} \pi \mathrm{SO}$ ). The signal level was chosen to be approximately just above the homophasic threshold in the model ( $60 \mathrm{~dB}$ ). The correlogram is plotted as a function of internal delay for auditory filters between 125 and $2000 \mathrm{~Hz}$. The stimulus was first filtered through a gammatone filter bank, transduced by an auditory hair-cell model (Meddis et al., 1990) and then the cross-product between corresponding filters at the two sides calculated. $\boldsymbol{D}, \boldsymbol{E}$, Center column represents the summary cross-correlation functions obtained by averaging across the $300 \mathrm{~Hz}-800 \mathrm{~Hz}$ frequency range. $\boldsymbol{D}$, Summary cross-correlations for the column on the left (NO). E, Summary cross-correlations for the right column ( $\mathrm{N} \pi$ ). $\boldsymbol{A}-\boldsymbol{H}$, Black represents noise alone, red represents homophasic conditions (NOSO and $\mathrm{N} \pi \mathrm{S} \pi$ ), and green represents antiphasic conditions ( $N O S \pi$ and $\mathrm{N} \pi \mathrm{SO}$ ).

same best frequency distribution as recorded in cortex. Following this, the best ITD distributions were not significantly different (Fig. 3A). As in the IC, most units were found to be tuned to the contralateral hemifield (Jiang et al., 1997b; McAlpine et al., 2001).

Distribution of $\mathrm{P}$ - and N-type thresholds The distribution of P- and N-type thresholds differed greatly between the stimulus conditions and somewhat from the previous data from the guinea pig IC (Jiang et al., 1997a). In cortex, the greatest differences were between the antiphasic conditions: the NOS $\pi$ condition yielded far more N-type than P-type thresholds (89\% N-type), whereas the reverse was true for the $\mathrm{N} \pi \mathrm{S} 0$ condition (90\% P-type). In the IC, the pattern was similar, but the differences were less extreme (53\% N-type for NOS $\pi$ and $60 \%$ P-type for $\mathrm{N} \pi \mathrm{S} 0$ ). The present results are more similar to those observed by Asadollahi et al. (2010) in the IC of the barn owl where responses were measured to stimuli presented at the unit's best or worst ITD

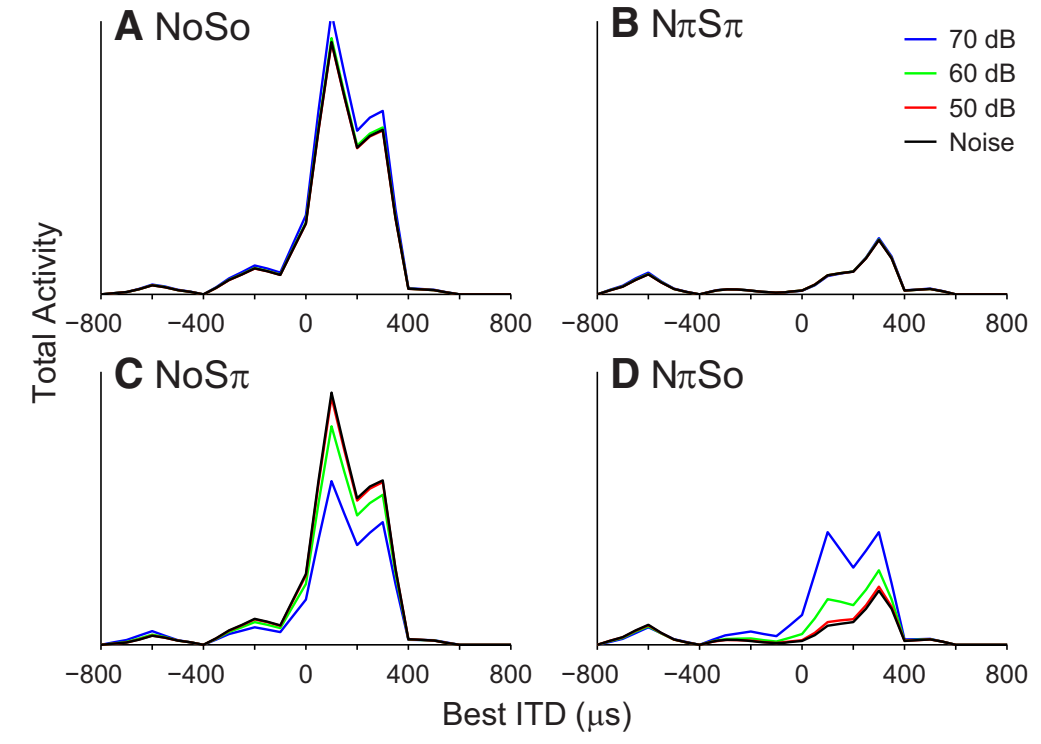

Figure 13. Output of a weighted binaural cross-correlation model for four signal levels of each condition. $A-D$, Average activity level for a population of neurons with a best frequency between 300 and $800 \mathrm{~Hz}$ distributed according to their best ITD (along the ordinate). The weighting function applied here reflects the distribution of best noise ITDs shown in Figure 3. Black line indicates the noise alone situation. The three other signal levels were chosen to match those presented in Figure 10 and represent signal levels around the detection threshold of each condition. 
rather than interaural phase differences of 0 or $\pi$ as in the current study. The responses to BMLD stimuli in cortex are even closer to the predictions from the neurons' rate-ITD functions (Jiang et al., 1997a, b; Palmer et al., 2000) than those from the IC (see Model of coincidence detection).

\section{Binaural masked thresholds and BMLDs}

The BMLD is determined by comparing the thresholds for homophasic and antiphasic stimulus conditions (e.g., NOSO vs $\operatorname{NOS} \pi$ ). There was a high proportion of units that only provided one of these thresholds, so intraneuron BMLDs could only be calculated for a few neurons. Among these intraneuron BMLDs, there was a high proportion of either very large, or negative, BMLDs. These were caused by units being relatively insensitive to one of the conditions and thus not representing the lowest threshold across units. It would therefore seem unlikely that psychophysical BMLDs are based on intraneuronal comparisons.

Instead, psychophysical BMLDs may be based on the most sensitive neurons for each condition (Jiang et al., 1997a), as suggested by the "lower envelope principle" (Parker and Newsome, 1998). This principle has been confirmed in various sensory modalities. It is supported by data on binaural discrimination (Skottun et al., 2001; Shackleton et al., 2003), tone detection and forward masking (Young and Barta, 1986; Relkin and Pelli, 1987; Relkin and Turner, 1988) in audition, as well as in the visual (Parker and Hawken, 1985; Bradley et al., 1987; Newsome et al., 1989; Hawken and Parker, 1990) and somatosensory modalities (Mountcastle et al., 1972; Johansson and Vallbo, 1979; Vallbo, 1995). Here, we calculated the 25th percentile of thresholds rather than taking the lowest threshold as in the original formulation of the lower envelope principle. The 25th percentile is likely to be a more reliable statistic given the relatively small number of measurements.

The relative numbers and values of $\mathrm{P}$ - and $\mathrm{N}$-type thresholds differed dramatically between different stimulus conditions (see earlier discussion; Figs. 5 and 6), suggesting that the response type that provides the lowest threshold for each condition is the one used to yield the psychophysical BMLD.

The population BMLDs obtained here $(\mathrm{N} 0 \mathrm{~S} 0-\mathrm{N} 0 \mathrm{~S} \pi=10 \mathrm{~dB}$; $\mathrm{N} \pi \mathrm{S} \pi-\mathrm{N} \pi \mathrm{S} 0=22 \mathrm{~dB}$ ) are comparable with psychophysical results (12-15 dB) (Durlach and Colburn, 1978), although, in animals, smaller behavioral BMLDs are often found (Wakeford and Robinson, 1974; Hine et al., 1994). In the IC, smaller BMLDs were measured (Jiang et al., 1997a, b; Palmer et al., 2000; Lane and Delgutte, 2005). The difference between IC and cortex appears to be due to lower antiphasic thresholds and higher homophasic thresholds.

\section{Model of coincidence detection}

Many models of binaural processing have been developed over the last 40 years (Colburn and Durlach, 1978; Stern and Trahiotis, 1995; for review, see Colburn, 1996); most are based on the coincidence-detection network proposed by Jeffress (1948). Previous data in the IC have been found to match this model well (Jiang et al., 1997b; Palmer et al., 2000). We used a binaural coincidence detection model (Figs. 12 and 13; see Materials and Methods) with the same signal levels as those used to compute the measured population responses across best ITD shown in Figure 10.

The cross-product model is consistent with the current data. The model predicts that adding the $S \pi$ signal to the N0 noise in the NOS $\pi$ condition causes a desynchronization of the inputs to the coincidence-detecting neurons. This should cause a decrease in the firing rate of neurons with small best ITDs, which are well driven by the N0 noise, as was observed in both the model and the data (Figs. $10 C$ and $13 C$ ). This explains the high proportion of N-type thresholds observed for the N0S $\pi$ condition (Fig. $6)$. By contrast, addition of the S0 signal to the $\mathrm{N} \pi$ noise in the $\mathrm{N} \pi \mathrm{S} 0$ condition would cause an increase in the synchronization of the inputs to the coincidence detecting neurons. As a result, the firing rate of neurons with small best ITDs, which are poorly driven by $\mathrm{N} \pi$ noise, should increase (Figs. $10 D$ and $13 D$ ), predicting a high proportion of P-type thresholds in the $\mathrm{N} \pi \mathrm{S} 0$ condition, in line with the data (Fig. 6). The model predicts the maximum effect when the best ITD or worst ITD of the neuron matches the interaural phase of the noise (Fig. 11).

The model predicted a small increase in activity with increasing signal level for the homophasic ( $\mathrm{N} 0 \mathrm{~S} 0$ and $\mathrm{N} \pi \mathrm{S} \pi$ ) conditions due to increased overall numbers of spikes at the input to the coincidence detectors. The measured activity for both the NOSO and $\mathrm{N} \pi \mathrm{S} \pi$ conditions reflected this pattern at intermediate signal levels. However, the model was not designed to predict the observed nonmonotonic behavior at signal levels $\sim>80 \mathrm{~dB}$ SPL (as in Fig. 9).

The model used to generate these cross-products is a generic one, optimized for modeling psychophysical rather than physiological data. It is remarkable, therefore, that such an unspecialized model is capable of predicting the real physiological data so well near threshold. As concluded in previous work (Jiang et al., 1997b; Palmer et al., 2000), this demonstrates that even a simple cross-product model is consistent with empirically measured neuronal processing of BMLDs.

In conclusion, we find that the response to BMLD stimuli is similar in IC and cortex. Signals are detected from increases or decreases in the binaural cross-product, and the psychophysical detection threshold is based on the lowest neural thresholds across the population.

\section{Notes}

Supplemental material for this article is available at http://www.ihr.mrc. ac.uk/projects/matlab/binaural_toolbox (Michael Akeroyd's Binaural toolbox for MATLAB). This material has not been peer reviewed.

\section{References}

Asadollahi A, Endler F, Nelken I, Wagner H (2010) Neural correlates of binaural masking level difference in the inferior colliculus of the barn owl (Tyto alba). Eur J Neurosci 32:606-618. CrossRef Medline

Bradley A, Skottun BC, Ohzawa I, Sclar G, Freeman RD (1987) Visual orientation and spatial frequency discrimination: a comparison of single neurons and behavior. J Neurophysiol 57:755-772. Medline

Bullock DC, Palmer AR, Rees A (1988) Compact and easy-to-use tungstenin-glass microelectrode manufacturing workstation. Med Biol Eng Comput 26:669-672. CrossRef Medline

Buunen TJ, Rhode WS (1978) Responses of fibers in the cat's auditory nerve to the cubic difference tone. J Acoust Soc Am 64:772-781. CrossRef Medline

Caird D, Palmer A, Rees A (1991) Binaural masking level difference effects in single units of the guinea pig inferior colliculus. Hearing Res 57:91-106. CrossRef Medline

Colburn HS (1977) Theory of binaural interaction based on auditory-nerve data: II. Detection of tones in noise. J Acoust Soc Am 61:525-533. CrossRef Medline

Colburn HS (1996) Computational models of binaural processing. In: Auditory computation (Hawkins HL, McMullen TA, Popper AN, Fay RR, eds), pp 332-400. New York: Springer.

Colburn HS, Durlach NI (1978) Models of binaural interaction. In: Handbook of perception, Vol IV, Hearing (Carterette EC, Friedman MP, eds), pp 467-518. New York: Academic.

Durlach NI, Colburn HS (1978) Binaural phenomena. In: Handbook of perception, Vol IV, Hearing (Carterette EC, Friedman MP, eds), pp 365466. New York: Academic. 
Franks NP, Lieb WR (1994) Molecular and cellular mechanisms of general anaesthesia. Nature 367:607-614. CrossRef Medline

Goldberg JM, Brown PB (1969) Response of binaural neurons of dog superior olivary complex to dichotic tonal stimuli: some physiological mechanisms of sound localization. J Neurophysiol 32:613-636. Medline

Green DM, Swets JA (1966) Signal detection theory and psychophysics. New York: Wiley.

Greene NT, Anbuhl KL, Williams W, Tollin DJ (2014) The acoustical cues to sound location in the guinea pig (Cavia porcellus). Hearing Res 316:1-15. CrossRef Medline

Hara K, Harris RA (2002) The anesthetic mechanism of urethane: the effects on neurotransmitter-gated ion channels. Anesth Analg 94:313-318. CrossRef Medline

Hawken MJ, Parker AJ (1990) Detection and discrimination mechanisms in the striate cortex of the old-world monkey. In: Vision: coding and efficiency (Blakemore C, ed), pp 103-116. Cambridge: Cambridge UP.

Hine JE, Martin RL, Moore DR (1994) Free-field binaural unmasking in ferrets. Behav Neurosci 108:196-205. CrossRef Medline

Hirsh IJ (1948a) Binaural summation and interaural inhibition as a function of the noise level of the masking noise. Am J Psychol 56:205-213. Medline

Hirsh IJ (1948b) The influence of interaural phase on interaural summation and inhibition. J Acoust Soc Am 20:536-544. CrossRef

Jeffress LA (1948) A place theory of sound localization. J Comp Psychol 44:35-39. Medline

Jiang D, McAlpine D, Palmer AR (1997a) Detectability index measures of binaural masking level difference across populations of inferior colliculus neurons. J Neurosci 17:9331-9339. Medline

Jiang D, McAlpine D, Palmer AR (1997b) Responses of neurons in the inferior colliculus to binaural masking level difference stimuli measured by rate-versus-level functions. J Neurophysiol 77:3085-3106. Medline

Johansson RS, Vallbo ÅB (1979) Detection of tactile stimuli: thresholds of afferent units related to psychophysical thresholds in the human hand. J Physiol 297:405-422. Medline

Lane CC, Delgutte B (2005) Neural correlates and mechanisms of spatial release from masking: single-unit and population responses in the inferior colliculus. J Neurophysiol 94:1180-1198. CrossRef Medline

Langford TL (1984) Responses elicited from medial superior olivary neurone by stimuli associated with binaural masking and unmasking. Hearing Res 15:39-50. CrossRef Medline

Lee CC, Middlebrooks JC (2013) Specialization for sound localization in fields A1, DZ, and PAF of cat auditory cortex. J Assoc Res Otolaryngol 14:61-82. CrossRef Medline

Licklider JCR (1948) The influence of interaural phase relations upon the masking of speech by white noise. J Acoust Soc Am 20:150-159. CrossRef

MacDonald RL, Rogers CJ, Twyman RE (1989) Barbiturate regulation of kinetic properties of the GABAA receptor channel of mouse spinal neurones in culture. J Physiol 417:483-500. Medline

Macmillan NA, Creelman CD (2005) Detection theory: a user's guide, Ed 2. Mahwah, New Jersey: Lawrence Erlbaum.

McAlpine D, Jiang D, Palmer AR (1996) Binaural masking level differences in the inferior colliculus. J Acoust Soc Am 100:490-503. CrossRef Medline

McAlpine D, Jiang D, Palmer AR (2001) A neural code for low-frequency sound localisation in mammals. Nat Neurosci 4:396-401. CrossRef Medline

Meddis R, Hewitt MJ, Shackleton TM (1990) Implementation details of a computation model of the inner hair-cell auditory-nerve synapse. J Acoust Soc Am 87:1813. CrossRef

Mountcastle VB, LaMotte RH, Carli G (1972) Detection thresholds for stimuli in humans and monkeys: comparison with threshold events in mechanoreceptive afferent nerve fibers innervating the monkey hand. J Neurophysiol 35:122-136. Medline

Newsome WT, Britten KH, Movshon JA (1989) Neuronal correlates of a perceptual decision. Nature 341:52-54. CrossRef Medline

Palmer AR, Jiang D, McAlpine D (2000) Neural responses in the inferior colliculus to binaural masking level differences created by inverting the noise in one ear. J Neurophysiol 84:844-852. Medline

Palmer AR, Shackleton TM, Sumner CJ, Zobay O, Rees A (2013) Classification of frequency response areas in the inferior colliculus reveals continua not discrete classes. J Physiol 591:4003-4025. CrossRef Medline

Parker A, Hawken M (1985) Capabilities of monkey cortical cells in spatialresolution tasks. J Opt Soc Am A 2:1101-1114. CrossRef Medline

Parker AJ, Newsome WT (1998) Sense and the single neuron: probing the physiology of perception. Annu Rev Neurosci 21:227-277. CrossRef Medline

Relkin EM, Pelli DG (1987) Probe tone thresholds in the auditory nerve measured by two-interval forced-choice procedures. J Acoust Soc Am 82:1679-1691. CrossRef Medline

Relkin EM, Turner CW (1988) A reexamination of forward masking in the auditory nerve. J Acoust Soc Am 84:584-591. CrossRef Medline

Sakitt B (1973) Indices of discriminability. Nature 241:133-134. CrossRef Medline

Shackleton TM, Skottun BC, Arnott RH, Palmer AR (2003) Interaural time difference discrimination thresholds for single neurons in the inferior colliculus of guinea pigs. J Neurosci 23:716-724. Medline

Skottun BC, Shackleton TM, Arnott RH, Palmer AR (2001) The ability of inferior colliculus neurons to signal differences in interaural delay. Proc Natl Acad Sci U S A 98:14050-14054. CrossRef Medline

Sterbing SJ, Hartung K, Hoffmann KP (2003) Spatial tuning to virtual sounds in the inferior colliculus of the guinea pig. J Neurophysiol 90: 2648-2659. CrossRef Medline

Stern RM, Trahiotis C (1995) Models of binaural interaction. In: Hearing (Moore BCJ, ed), pp 347-386. San Diego: Academic.

Sumner CJ, Palmer AR (2012) Auditory nerve fibre responses in the ferret. Eur J Neurosci 36:2428-2439. CrossRef Medline

Ter-Mikaelian M, Sanes DH, Semple MN (2007) Transformation of temporal properties between auditory midbrain and cortex in the awake Mongolian gerbil. J Neurosci 27:6091-6102. CrossRef Medline

Tollin DJ, Koka K, Tsai JJ (2008) Interaural level difference discrimination thresholds for single neurons in the lateral superior olive. J Neurosci 28:4848-4860. CrossRef Medline

Vallbo $\AA$ B (1995) Single-afferent neurons and somatic sensation in humans. In: The cognitive neurosciences (Gazzaniga MS, ed), pp 237-252. Cambridge, MA: Massachusetts Institute of Technology.

Wakeford OS, Robinson DE (1974) Detection of binaurally masked tones by the cat. Journal of the Acoustical Society of America 56:952-956.

Wallace MN, Rutkowski RG, Palmer AR (2000) Identification and localisation of auditory areas in guinea pig cortex. Exp Brain Res 132:445-456. CrossRef Medline

Yin P, Fritz JB, Shamma SA (2014) Rapid spectrotemporal plasticity in primary auditory cortex during behavior. J Neurosci 34:4396-4408. CrossRef Medline

Yin TC, Kuwada S (1983) Binaural interaction in low-frequency neurons in inferior colliculus of the cat: III. Effects of changing frequency. J Neurophysiol 50:1020-1042. Medline

Young ED, Barta PE (1986) Rate responses of auditory nerve fibers to tones in noise near masked threshold. J Acoust Soc Am 79:426-442. CrossRef Medline 